\title{
Robust Hybrid Algorithm of PSO and SOCP for Grating Lobe Suppression and against Array Manifold Mismatch
}

\author{
Hailin LI, Jialing LIU, Jie SUN, Aihua CAO, Can JIN, Jianjiang ZHOU \\ College of Electronic and Information Engineering, Nanjing University of Aeronautics and Astronautics, \\ No. 29 Jiangjun Avenue, 211100 Nanjing, China \\ nuaalhls@nuaa.edu.cn, liujialing1995@nuaa.edu.cn,18020167295@163.com,15150503571@163.com, \\ jc_sakura@163.com,zjjee@nuaa.edu.cn
}

Submitted April 4, 2018 / Accepted September 18, 2018

\begin{abstract}
Based on Particle Swarm Optimization (PSO) and Second-Order Cone Programming (SOCP) algorithm, this paper proposes a hybrid optimization method to suppress the grating lobes of sparse arrays and improve the robustness of array layout. With the peak side-lobe level (PSLL) as the objective function, the paper adopts the particle swarm optimization as a global optimization algorithm to optimize the elements' positions, the convex optimization as a local optimization algorithm to optimize the elements' weights. The effectiveness of the grating lobes suppression (as low as -32.13 dB) by this method is illustrated through its application to the sparse linear array when the actual steering vector is known. To enhance the robustness of the optimized array, a rebuilt robust convex optimization model is adopted in the optimization of both array excitations and layout. When the array manifold mismatch error is $1 \mathrm{~cm}$, the PSLL by the robust algorithm can be compressed to $-27 d B$, compared to that of $-24 d B$ by the ordinary optimization. Results of a set of representative numerical experiments show that the algorithm proposed in this paper can obtain a more robust array layout and matched elements' weight coefficients to avoid the huge degradation of the array pattern performance in the presence of array manifold mismatch errors. The good performance of pattern synthesis demonstrates the effectiveness of the proposed robust algorithm.
\end{abstract}

\section{Keywords}

Particle Swarm Optimization (PSO), Second-Order Cone Programming (SOCP), array manifold mismatch, grating lobes suppression, hybrid algorithm

\section{Introduction}

In recent years, array antennas have been widely used in wireless mobile communication, radar, navigation of aircraft and many other fields. However, the number of the array elements and array size limitation often result in the complexity and high cost of antenna system. Therefore, sparse array antenna is developed to solve these problems. Sparse array refers to non-uniformly-spaced array where both the spacing and the position of the array elements are distributed irregularly and randomly within a certain range. Compared to conventional arrays, sparse arrays have the advantages of narrower beam width, lower cost and higher resolution [1-3]. However, the defects of the sparse arrays such as the grating lobes and the high side-lobe level, are the critical issues to be solved in the research of sparse array [4], [5]. Moreover, in practical application, the jitter of array elements and mutual coupling between adjacent elements often cause array manifold mismatch, which leads to an unavoidable degradation of the array performance. Thus, designing a robust array against manifold mismatch has become a key technical issue in the research of sparse array in recent years [6], [7].

Much research has been done to solve the high sidelobe level (SLL) problem created by sparse arrays and the relevant studies can be divided into two main broad categories: intelligent algorithms and convex optimization.

With regard to intelligent optimization algorithms, genetic algorithm (GA) and particle swarm optimization (PSO) have been widely used in the design of sparse array. To suppress the peak side-lobe level (PSLL) of sparse concentric ring arrays, a modified real genetic algorithm (MGA) was proposed to optimize the grid ring radii in [8], which made an effort to improve the searching ability. The simulations of 6 grid rings and 8 grid rings illustrated the effectiveness of MGA, but only improved $0.51 \mathrm{~dB}$ in PSLL compared to [9]. Jiang et al. presented an improved integer genetic algorithm (IIGA) to meet the needs of sparse array design with thousands of array elements [10]. The modified algorithm had a PSLL of $0.75 \mathrm{~dB}$ improvement compared to WD method [11], while the array elements were reduced by $6.85 \%$. However, this approach was time-consuming for large arrays. Matthew et al. used a genetic algo- 
rithm to optimize the locations of antennas in a linear sparse array under the constraint of their physical size [12], [13]. The coefficients of elements and positions of the array were the decision variables in [14], [15] to achieve a lower side-lobe level. GA-based optimization and dynamic constrained multi-objective evolutionary algorithm (DCMOEA) were designed for planar arrays and linear sparse arrays respectively. The PSLL of sparse linear array of 19 elements with an overall aperture of $18.66 \lambda$ ( $\lambda$ is the wavelength) could be reduced to $-14.01 \mathrm{~dB}$. However, the array pattern obtained by these methods mentioned above is far from the best because of the defect that the single evolutionary algorithms always plunged into the local optimal solution. In order to suppress the grating lobes of sparse arrays, PSO was used to obtain array layout under the constraint of minimum and maximum spacing of the adjacent elements [16]. Although the performance of the optimized array pattern is fine, the convergence speed was poor. The same idea was used in [17], [18], in which only the positions of array elements were optimized. These methods were effective to obtain the lowest PSLL as well as a narrower beam width, but the pattern synthesis of the whole space was not allowed, which limited the extensive use of them. Li et al. considered using genetic algorithm to optimize the phase and amplitude distribution of the theoretical linear array [19], but the positions of the array elements were obtained in the global optimization. Similarly, in the studies of [20], [21], PSO and a modified adaptive PSO algorithm (MPSO) were used for the design of the sparse array without the consideration of the complete match of the elements' positions and weights.

In addition to the intelligent algorithm mentioned above, a variety of antenna array pattern synthesis problem have been converted into convex form [22], [23], which can be handled by a convex problem solver such as Sedumi and CVX [24], [25] with great efficiency. Benjamin tried to minimize the number of radiating elements of sparse arrays under the constraint of the specified pattern requirements by sequential convex optimization, which included the transformation from a non-convex optimization problem to a sequence of essentially convex one [26]. Likewise, in [27-31], the optimizations of the sparse array were all converted into the standard convex form and solved by Sedumi in Matlab easily. With these methods, improved performance has been achieved, characterized by a final PSLL almost to $-30 \mathrm{~dB}$, with 13 array elements and an aperture of $9.49 \lambda$. However, the conversion mentioned above requires the weighted $l_{1}-$ norm as close as to $l_{0}-$ norm, which was difficult to achieve. Zhao et al. proposed a combined new optimization algorithm which used genetic algorithm to get elements' positions and applied convex method to optimize elements' weight coefficients [32]. Under the constraint of main lobe width, the best PSLL for a 25 -elements array over an aperture of $50 \lambda$ was optimized to $-13.86 \mathrm{~dB}$. But this approach could only choose positions at specific locations and optimize the amplitude of the elements' weights without consideration of the phase, which reduced the freedom of optimization variables. The locations of the array elements and the coefficients were taken as joint variables to minimize the grating lobes of sparse circular array through modified genetic algorithm in [33], and then the second-time optimization was conducted via second-order cone programming (SOCP) to get the perfect match between these two variables, which further reduced the PSLL. The study took into consideration the combined characteristics of these two optimized variables, but their perfect match was only applied in the last step in SOCP.

The papers mentioned above all assumed that the steering vector of an antenna array was definite and ignored the manifold mismatch. However, in practical application, the actual array manifold may differ from the ideal value due to the mutual coupling and position errors. To achieve the robustness of the optimized array, many robust optimizations have been done to enhance the robust performance and avoid expensive and time-consuming calibrations in the practical application of array antenna. Interval analysis (IA) was widely used in the robust adaptive beam forming algorithm in [34-38]. In these studies, given the maximum errors on the amplitude coefficients, IA-based tool was used to effectively achieve the ideal synthesis of robust antenna arrays. But the phase coefficients were not considered. Aboulnasr et al. proposed an iterative sequential quadratic programming (SQP) to handle the mismatch problem [39]. The core idea was to predicate the difference between the presumed and actual steering vectors and then use it to correct the errors of vectors without assuming the norm of the mismatch parameters. With regard to the constant constrained error, the worst-case performance optimization was proposed in [40] to enhance the robustness of the optimized conformal array and decrease the PSLL simultaneously. Just like [41], the convexity of the robust problem was fully taken into account to make the solutions simple and convenient, which increased the extendibility of the presented method. The two optimization criteria of $l_{2}$ regularization and $l_{1}$ regularization were proposed in [42], [43] to synthesize array pattern with low PSLL and enhance the robustness of array manifold vectors. The problem of robust array pattern synthesis was equivalently transformed into convex form, which was handled by SOCP solver. However, the optimization of the array layout was not considered by the author. Liu et al. presented a robust sparse optimization for linear array against manifold vectors uncertainty, which was also based on the iterative convex optimization [44]. To obtain a robust array pattern performance, the transformation of non-convex constraints was widely used in the above methods and the reconstructed array showed the validity.

Unfortunately, the grating lobes suppression algorithm, which takes the robustness of the designed array against array manifold mismatch into consideration, has hardly been investigated. Most of the studies focus only on one aspect, which is not enough for the practical applications of the sparse array.

In this research, a robust hybrid optimization method 
of PSO and SOCP against array manifold mismatch is proposed for suppressing the grating lobes of the sparse array, with full consideration of the advantages and disadvantages of the algorithms mentioned above, where PSO is adopted to optimize the elements' positions and convex optimization is used for the weights to guarantee the complete match of these two variables. Compared with GA, PSO has some advantages such as simple operation and easy implementation. Besides, with the unique feature of SOCP that a local optimal solution is the same as the global optimal, a combination of PSO and SOCP is proposed. Because the perturbations of the elements' positions affect the array manifold and the performance of the grating lobes suppression is particularly sensitive to the array manifold mismatch, it is urgent to improve the robustness of the optimized array. To solve this problem, array manifold mismatch errors are introduced in local optimization algorithm and the robust convex cone models are rebuilt in this research. Several simulation results prove the correctness and effectiveness of the proposed hybrid algorithm.

This paper is composed of five sections. Some key studies in the field of sparse arrays are reviewed in Sec. 1 and the question to be addressed by the present study is pointed out. The model of optimization and the fundamental formula deduction are provided in Sec. 2. In Sec. 3, the optimization algorithm is presented, in which the form of robust convex cone is an improved version of a general form, taking the array manifold mismatch errors into consideration. In Sec. 4, the effectiveness of this hybrid algorithm is shown by detailed simulation results. Finally, Section 5 presents the conclusions.

\section{Optimization Model}

Consider a sparse linear array of $N$ isotropic elements, as shown in Fig. 1, the distance between the $n$th element and the origin of the reference coordinate system is defined as $d_{n}$ for $n=1,2, \ldots, N$. To keep the array aperture fixed as $L$, the first and last element are located at $d_{1}=0$ and $d_{L}=L$, respectively. The total electric field intensity of far field is given by the following expression

$$
E(\theta)=\sum_{n=1}^{N} w_{n}{ }^{*} \mathrm{e}^{\mathrm{j} k d_{n} \sin \theta}
$$

where $w_{n}$ is the $n$th element's complex weight coefficient, $(\cdot)^{*}$ is the complex conjugate operator, $k=2 \pi / \lambda$ is the propagation coefficient of the free space with the wavelength $\lambda$, and $\theta$ is the angular direction with respect to the array axis.

Given the constraint of PSLL, the objective function can be expressed as

$$
\begin{array}{ll}
\min & \max _{\Omega_{S}}\left|E\left(\theta_{m}\right)\right|, \quad m=1, \cdots, M \\
\text { s.t. } & E\left(\theta_{0}\right)=1 \\
& 0<x_{i}<L, \quad i=1, \cdots, N-2
\end{array}
$$

where PSLL is defined as $P S L L=\max \left|E\left(\theta_{m}\right) / \max E(\theta)\right|$,

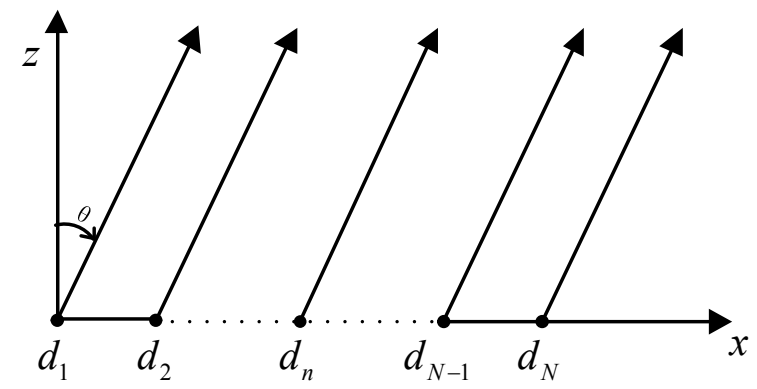

Fig. 1. Diagram of a sparse linear array.

$E\left(\theta_{0}\right)$ and $E\left(\theta_{m}\right)$, for $m=1, \ldots, M$, are the electric field intensity in the direction of the main radiation direction $\theta_{0}$ and $m$ th side-lobe angle $\theta_{m}$ in side-lobe region $\Omega_{\mathrm{S}}$, respectively. $x_{i}=d_{i+1}$ for $i=1, \ldots, N-2$ is the element positions waiting to be optimized.

When the array layout is fixed, Equation (2) can be expressed as

$$
\begin{array}{ll}
\min & \max _{\Omega_{S}}\left|\mathbf{w}^{\mathrm{H}} \mathbf{b}_{m}\right|, \quad m=1, \cdots, M \\
\text { s.t. } & \mathbf{w}^{\mathrm{H}} \mathbf{b}_{0}=1 \\
& 0<x_{i}<L, \quad i=1, \cdots, N-2
\end{array}
$$

where $\mathbf{w}=\left[w_{1}, \ldots, w_{N}\right]^{\mathrm{T}}$ is the complex excitation vector of the antenna array, and $\mathbf{b}_{m}=\mathbf{b}\left(\theta_{m}\right)=\left[1, \exp \left(\mathrm{j} k x_{1} \sin \theta\right), \ldots\right.$, $\left.\exp \left(\mathrm{j} k x_{N-2} \sin \theta\right), \quad \exp (\mathrm{j} k L \sin \theta)\right]^{\mathrm{T}}, \quad m=0, \quad 1, \ldots, M$ is the steering vector. $(\cdot)^{\mathrm{T}},(\cdot)^{\mathrm{H}}$ are the transpose operator and conjugate transpose operator, respectively.

\section{PSO-SOCP/RSOCP Algorithm}

The problem of (3) is a high dimensional non-linear problem, including the optimization of the array elements' positions and complex excitation weights, which can be optimized by PSO. But the high dimension of the problem will make the algorithm fall into a local optimal solution as well as a time-consuming graint. However, with the characteristic that a local optimal solution is the same as the global optimal solution, convex optimization can solve the problem perfectly. Thus, a hybrid method combining PSO algorithm and SOCP algorithm is proposed to get better optimal performance by reducing the dimension of particles to obtain the best solution for every particle. With this hybrid method, PSO is set as the global optimization algorithm to optimize the positions, and SOCP is set as the local optimization algorithm to optimize the complex weights with fixed array layout, respectively.

\subsection{SOCP Algorithm for the Fixed Array Layout}

Assume that the array element layout is fixed, $d_{n}, \forall n \in\{1, \ldots, N\}$ and $x_{i}, \forall i \in\{1, \ldots, N-2\}$ are constants. For the $m$ th side-lobe region angle $\theta_{m}$, the steering vector $\mathbf{b}_{m}$ is a constant vector. Then, the problem in (3) can be converted to a SOCP problem 


$$
\begin{array}{ll}
\min & \alpha \\
\text { s.t. } & \mathbf{b}_{0}^{\mathrm{H}} \mathbf{w}=1 \\
& \left|\mathbf{b}_{m}^{\mathrm{H}} \mathbf{w}\right| \leq \alpha, m=1, \cdots, M
\end{array}
$$

where $\alpha$ is a real and nonnegative variable. The constraints in (4) are equal to

$$
\begin{aligned}
& 1-\left(\begin{array}{ll}
0 & \mathbf{b}_{0}^{\mathrm{H}}
\end{array}\right) \mathbf{y}=1-\mathbf{b}_{0}^{\mathrm{H}} \mathbf{w} \in\{0\} \\
& \left(\begin{array}{l}
0 \\
0
\end{array}\right)-\left(\begin{array}{cc}
-1 & \mathbf{0}_{1 \times N} \\
0 & -\mathbf{b}_{m}^{\mathrm{H}}
\end{array}\right) \mathbf{y}=\left(\begin{array}{c}
\alpha \\
\mathbf{b}_{m}^{\mathrm{H}} \mathbf{w}
\end{array}\right) \in \operatorname{cone}^{2}, m=1, \cdots, M
\end{aligned}
$$

if $\mathbf{y}=\left(\alpha w_{1} \ldots w_{N}\right)^{\mathrm{T}}$. Then Equation (4) can be expressed as the standard SOCP form [23]

$$
\begin{array}{ll}
\max & \mathbf{a}^{\mathrm{T}} \mathbf{y} \\
\text { s.t. } & \mathbf{c}-\mathbf{A}^{\mathrm{T}} \mathbf{y} \in\{0\} \times \text { cone }_{1}^{2} \times \cdots \times \text { cone }_{M}^{2}
\end{array}
$$

where $\quad \mathbf{a}=\left(-1, \mathbf{0}_{1 \times N}\right)^{\mathrm{T}} \quad, \quad \mathbf{c}=\left(1, \mathbf{0}_{1 \times 2 M}\right) \quad$ and $\mathbf{A}=\left(\begin{array}{ccccccc}0 & -1 & 0 & \cdots & \cdots & -1 & 0 \\ \mathbf{b}_{0}^{*} & \mathbf{0}_{N \times 1} & -\mathbf{b}_{1}^{*} & \cdots & \cdots & \mathbf{0}_{N \times 1} & -\mathbf{b}_{M}^{*}\end{array}\right) \in \mathbb{C}^{(N+1) \times(2 M+1)}$

with $\mathfrak{R}$ and $\mathbb{C}$ being the set of real and complex scalars (or matrix), respectively.

With the excitation vector $\mathbf{w}$ optimized by SOCP optimization, PSO is applied to obtain the best position vector of the array elements by updating the particle positions, computing and comparing the value of fitness function, which is designed to evaluate the performance of the particles. Value of fitness function is defined as

$$
\text { fitness }=\max _{\Omega_{S}}\left|\mathbf{w}^{\mathrm{H}} \mathbf{b}_{m}\right|, \quad m=1, \cdots, M .
$$

Thus, the non-linear high dimensional optimization problem of (3) is converted to the combination of PSO and SOCP problem, taking advantages of these two optimization algorithms and reducing the probability of algorithms falling into a local optimal solution.

\subsection{Robust SOCP Algorithm for Mismatched Array Manifold}

Equation (4) can be seen as an optimization algorithm for grating lobes suppression without considering the manifold error. However, mismatches are found in many practical arrays for the perturbation of elements' positions, the discrepancy of directional function of array elements as well as mutual coupling between array elements. All these factors may change the steering vector $\mathbf{b}(\theta)$, resulting in a false solution. Thus, the robust SOCP(RSOCP) algorithm is proposed as follows.

Presume $\tilde{\mathbf{b}}(\theta)$ is the mismatched array manifold, $\mathbf{e}(\theta)$ is array manifold error, thus $\tilde{\mathbf{b}}(\theta)=\mathbf{b}(\theta)+\mathbf{e}(\theta)$, the error $\mathbf{e}(\theta)$ is always unknown but a threshold $\varepsilon$ can be given as $\|\mathbf{e}(\theta)\| \leq \varepsilon$, then $\left|\mathbf{b}^{\mathrm{H}}(\theta) \mathbf{w}\right|-\varepsilon\|\mathbf{w}\| \leq\left|\tilde{\mathbf{b}}^{\mathrm{H}}(\theta) \mathbf{w}\right| \leq\left|\mathbf{b}^{\mathrm{H}}(\theta) \mathbf{w}\right|+\varepsilon\|\mathbf{w}\|$. Therefore, with regard to robust optimization, the optimization problem of (4) can be written as the robust convex form as follows:

$$
\begin{array}{ll}
\min & \max \left(\left|\mathbf{b}_{m}^{\mathrm{H}} \mathbf{w}\right|+\varepsilon\|\mathbf{w}\|\right), m=1, \cdots, M \\
\text { s.t. } & \mathbf{b}_{0}^{\mathrm{H}} \mathbf{w}-\varepsilon\|\mathbf{w}\| \geq 1
\end{array}
$$

where $\left|\mathbf{b}_{m}{ }^{H} \mathbf{w}\right|+\varepsilon\|\mathbf{w}\|$ is the largest electric field intensity in the direction of side lobe $\theta_{m}$ of the mismatched array and $\mathbf{b}_{0}{ }^{\mathrm{H}} \mathbf{w}-\varepsilon\|\mathbf{w}\|$ is the smallest electric field intensity in the direction of the set radiation angle $\theta_{0}$. Similar to (4), when the position vector $\mathbf{x}$ is fixed, assume $\beta$ is a real and nonnegative variable, the problem in (8) can be converted to:

$$
\begin{array}{ll}
\min & \beta \\
\text { s.t. } & 1+\varepsilon\|\mathbf{w}\| \leq \mathbf{b}_{0}^{\mathrm{H}} \mathbf{w} \\
& \left|\mathbf{b}_{m}^{\mathrm{H}} \mathbf{w}\right|+\varepsilon\|\mathbf{w}\| \leq \beta, m=1, \cdots, M
\end{array}
$$

which is also a typical SOCP problem and its weights can be optimized by SOCP algorithm.

Then the fitness function of the RSOCP algorithm is re-defined as

$$
\text { fitness }=\frac{\max \left\|\mathbf{b}_{m}^{\mathrm{H}} \mathbf{w} \mid+\varepsilon\right\| \mathbf{w} \|, m=1, \cdots, M}{\left|\mathbf{b}_{0}^{\mathrm{H}} \mathbf{w}\right|-\varepsilon\|\mathbf{w}\|}
$$

which, taking into the consideration of error threshold $\varepsilon$, limits the optimal PSLL to a value that won't be worse than the fitness function value.

\subsection{The Procedure of PSO-SOCP/RSOCP Algorithm}

In this paper, the hybrid algorithm of PSO and SOCP/RSOCP takes the advantages of both algorithms. Because of its high efficiency and simplicity, PSO is applied as global optimization algorithm, and SOCP/RSOCP is applied as local optimization algorithm for its characteristic that the local optimal solution is the global optimal solution. All these features guarantee that the particles can search the whole space with the best local solution. The following are the detailed descriptions of the hybrid PSOSOCP/RSOCP algorithm.

1) Set the element number and aperture of the specific array model. Choose the angle sampling interval and the appropriate main lobe width of zero power point in accordance with the aperture.

2) Initialize the particle population and the speed of each particle. Use PSO to optimize the model in (2). Each particle's position vector represents the location of the array elements.

3) WHILE maximum number of cycles has not been reached, DO

a) Obtain weight coefficients of each array element by SOCP/RSOCP algorithm. 
b) Compute fitness values.

c) Update global optimal solution and historical optimal solution.

d) Update positions and velocities of the particles.

e) Increase the loop counter.

4) End cycle

5) Display the best results of pattern synthesis.

Figure 2 is the flowchart of the hybrid PSO-SOCP/RSOCP algorithm.

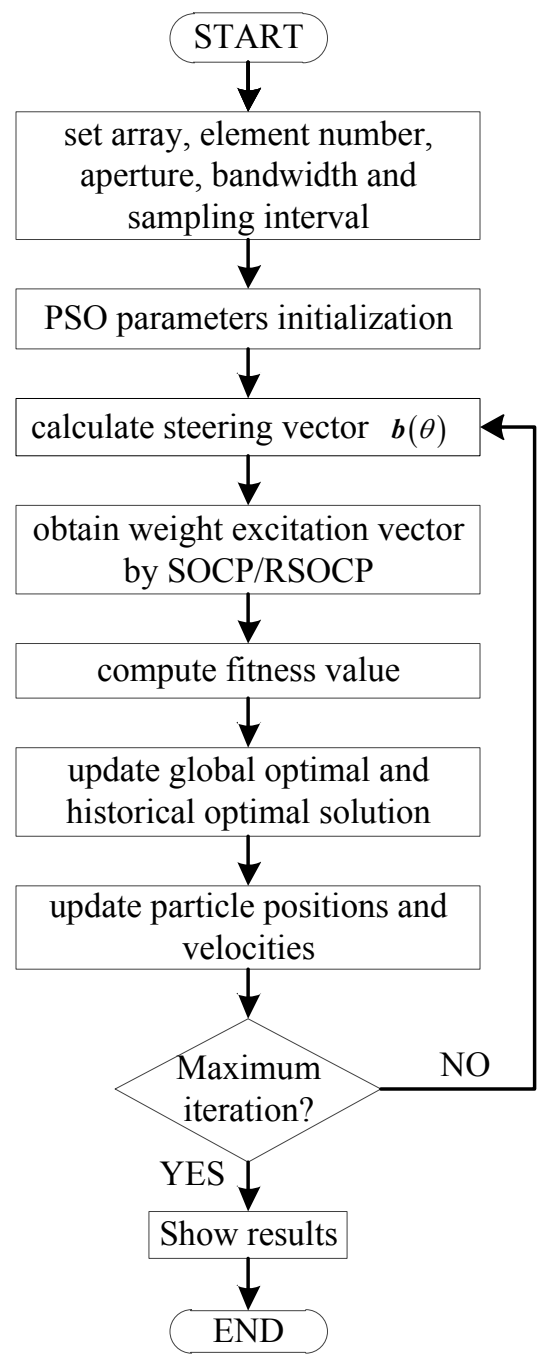

Fig. 2. Flowchart of $\mathrm{PSO}-\mathrm{SOCP} / \mathrm{RSOCP}$.

\section{Simulation Results}

In array signal processing, in order to suppress the grating lobes, the adjacent element spacing of the array should be less than or equal to a half wavelength. However, under the constraint of the limited elements number and array aperture, the array can only be sparsely populated. In order to describe sparse degree of the sparse array, the sparse ratio is defined as

$$
\eta=\left(1-\frac{M}{l}\right) \times 100 \%
$$

where $M$ is the number of the physical elements in the sparse array, $l$ is the number of the equivalent elements formed by half-wavelength interval array. Sparse ratio combines two factors: the number of elements and the aperture of the array. Therefore, the sparse ratio reflects the sparseness of the distributed array, that is, the larger of the sparse ratio, the sparser of the array.

In this section, a variety of simulations and comparisons are presented in order to illustrate the advantages of the proposed hybrid algorithm. Firstly, without array manifold perturbations, the proposed PSO-SOCP algorithm is compared with the previously published methods in terms of the ability to suppress the grating lobes (Figs. 3, 4). Then, new simulations and results are listed, in which RSOCP model proposed in Sec. 3.2, is adopted to modify and obtain much more robust elements' excitations (Figs. 5-7). Finally, the simulations of the RSOCP algorithm combined with PSO algorithm are presented, which optimize the weight vector as well as the position vector to obtain not only the robust weight coefficients but also the robust array layout (Figs. 8-11).

\subsection{Simulations of the Grating Lobes Suppression}

Assume a 50 element linear array with a fixed aperture of $5 \mathrm{~m}$ and a working wavelength of $0.06 \mathrm{~m}$. The main radiation direction of the pattern synthesis is set as $10^{\circ}$, and the width of the main lobe zero power points is set as $3^{\circ}$. Under these conditions, the equivalent number of elements with $d=\lambda / 2$ is 167 , thus the sparse ratio is $70 \%$ according to $(11)$.

The optimization model described above is a typical sparse array with a sparse ratio of $70 \%$. The parameters to be optimized are the positions and weight coefficients of the array elements. Simulations in the following verify the effectiveness of the proposed algorithm in terms of grating lobe suppression when there is no error in the steering vector of the actual array.

In order to suppress the grating lobes of the sparse model and improve the performance of directivity pattern, firstly, PSO is used according to the formula presented in (3). The basic parameters of PSO are given in Tab. 1 where $c_{1}$ and $c_{2}$ are personal and global learning coefficients, respectively.

Figure 3 shows the comparison of radiation patterns using the PSO algorithm, the secondary optimization algorithm by SOCP after PSO and hybrid PSO-SOCP algo-

\begin{tabular}{|l|c|}
\hline angle sampling interval & $0.2^{\circ}$ \\
\hline population size & 300 \\
\hline Iteration number & 200 \\
\hline learning coefficients & $c_{1}=c_{2}=1.4962$ \\
\hline
\end{tabular}

Tab. 1. Basic parameters of PSO. 
rithm proposed in this paper. PSO is run 30 times for independent trials, the best value of PSLL is $-14.58 \mathrm{~dB}$. With the secondary optimization by SOCP after PSO, PSLL can be suppressed to $-23.69 \mathrm{~dB}$, which is much lower than that of original PSO. The PSO-SOCP hybrid optimization algorithm proposed in this paper pushes the PSLL to $-32.13 \mathrm{~dB}$ while broadening the main lobe width by $2^{\circ}$, which is within the acceptable range and understandable for the reduction of the side lobes will result in a broadening of the main lobe. The optimization effect of the hybrid algorithm is much better than that of the PSO and the second optimization algorithm in the literature [33].

The better numerical values indicate that the idea of introducing convex optimization to get better weights with obtained array layout by PSO is indeed effective in the pattern synthesis of the sparse array.

To further discuss in detail the validity and reliability of the hybrid method, Figure 4 draws the convergence curves of the PSLL obtained by this algorithm for 30 independent trials. Already for the zeroth or the first iteration the fitness value is $-27 \mathrm{~dB}$, thus it is far below those of the compared methods. The hybrid algorithm of PSO and SOCP proposed in this paper can enhance the ability of each particle's optimal search in each iteration through reducing the dimension of the particles, preventing the particles to fall into local optimum. Given their respective contribution to global optimization and local optimization, combining PSO algorithm and SOCP algorithm can greatly enhance the optimization ability of the entire algorithm and achieve better grating lobe suppression effect.

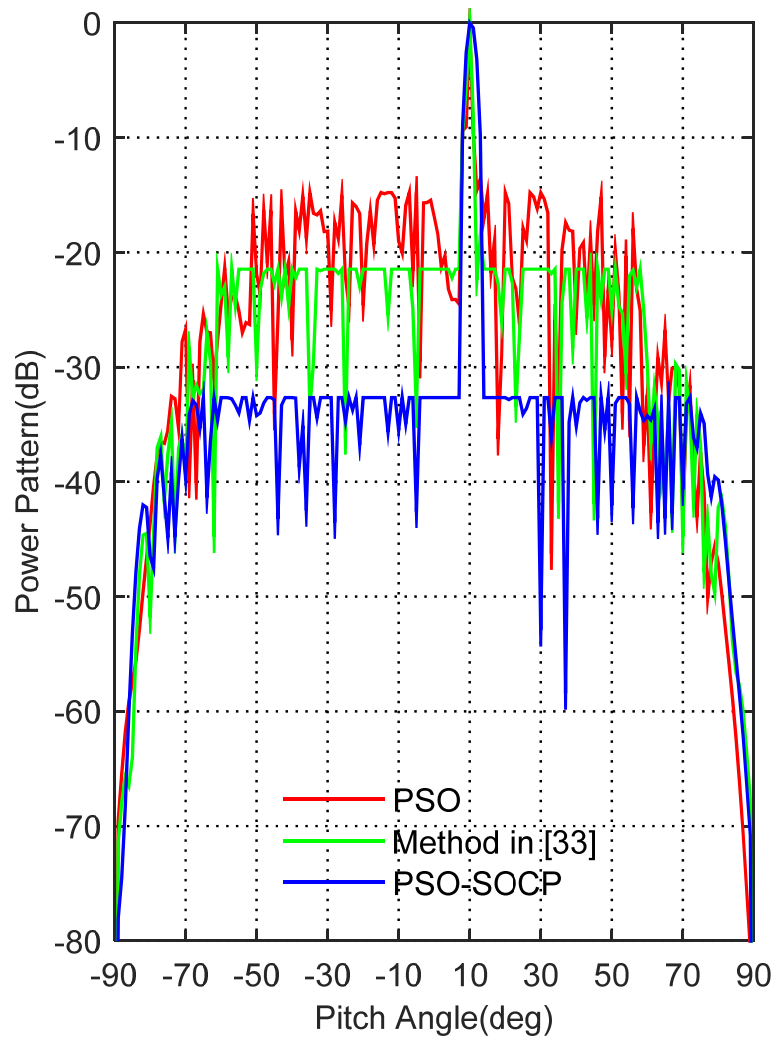

Fig. 3. Radiation patterns of three different methods.

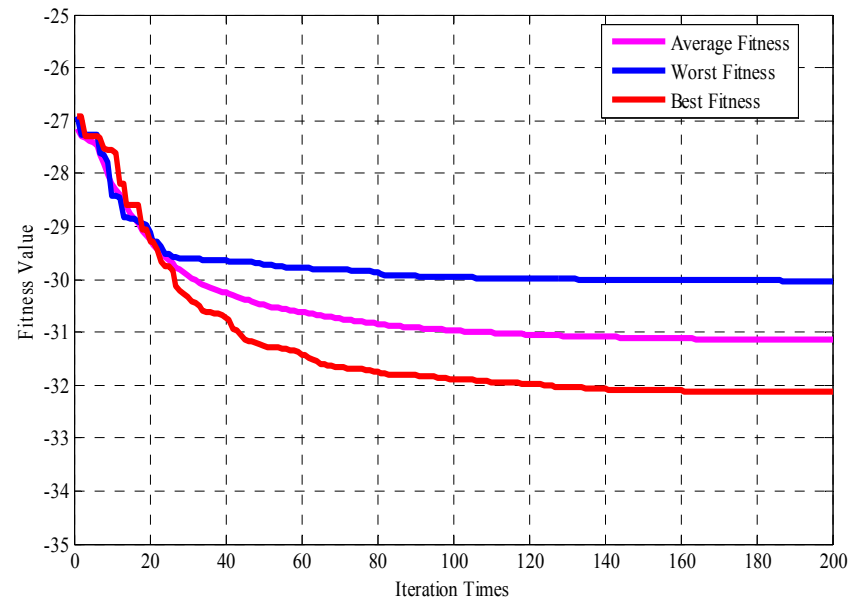

Fig. 4. Convergence curves of 30 independent trials.

Figure 4 shows that it tends to converge at about the 140th iteration, and the worst fitness is $-30.034 \mathrm{~dB}$ while the best fitness is $-32.13 \mathrm{~dB}$, which is better than the result of $-14.58 \mathrm{~dB}$ by PSO and $-23.69 \mathrm{~dB}$ by the method in literature [33]. And the best result of the proposed hybrid algorithm has improved $17.55 \mathrm{~dB}$ in PSLL compared with PSO, and $8.44 \mathrm{~dB}$ compared with the method in literature [33].

The above-mentioned statistics suggest that the proposed hybrid algorithm outperforms the other algorithms in the ability to suppress the grating lobes of the sparse array, which exhibits its superiority and efficiency.

\subsection{The Sensitivity Analysis of the Obtained PSO-SOCP Results}

The simulation results discussed above have testified the validity of the proposed hybrid algorithm. Those simulations, however, are conducted without considering the array manifold mismatch factor. In fact, the steering vector perturbation of the array can greatly reduce the performance of the array pattern, so the simulations of this section are performed under the condition that array manifold mismatch errors are true. The uncertainty for the location of the optimized array is considered in the simulations.

Using the PSO-SOCP algorithm proposed in this paper, the simulation in Sec. 4.1 has obtained the optimal array layout and excitation coefficient. Then the array element perturbation $(1 \mathrm{~mm}-5 \mathrm{~cm})$ was added and 10 independent trials were carried out.

Figure 5 shows the maximum threshold of the array manifold error functioning as position error. As can be seen from the figure, the array manifold error $\varepsilon$ increases as the position error increases. Since 10 statistical experiments were performed, the mean value, maximum value, and minimum value of the error threshold were approximately linear with the position error.

The PSLL change curves vs. position error are put forward in Fig. 6. Without position error, the PSO-SOCP hybrid optimization algorithm proposed in this paper pushes the PSLL to $-32.13 \mathrm{~dB}$. It can be seen from Fig. 6 


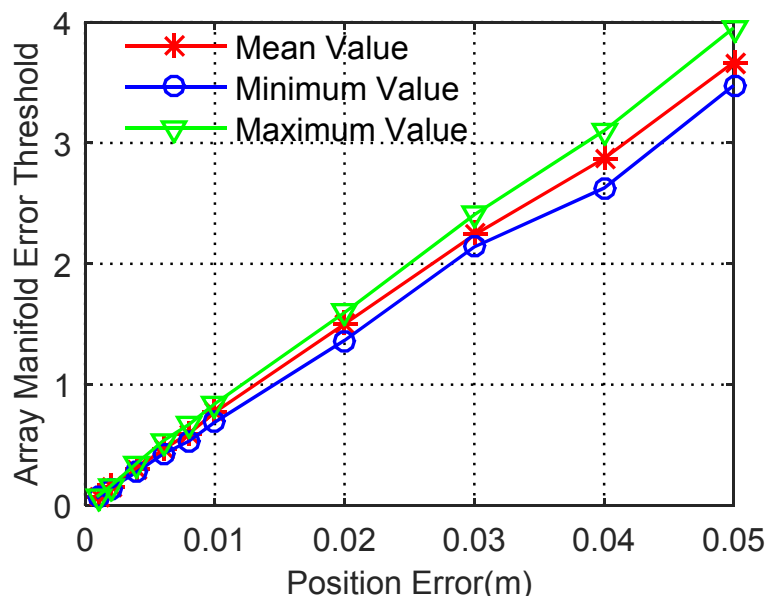

Fig. 5. Array manifold error threshold $\varepsilon$ change curves vs. position errors.

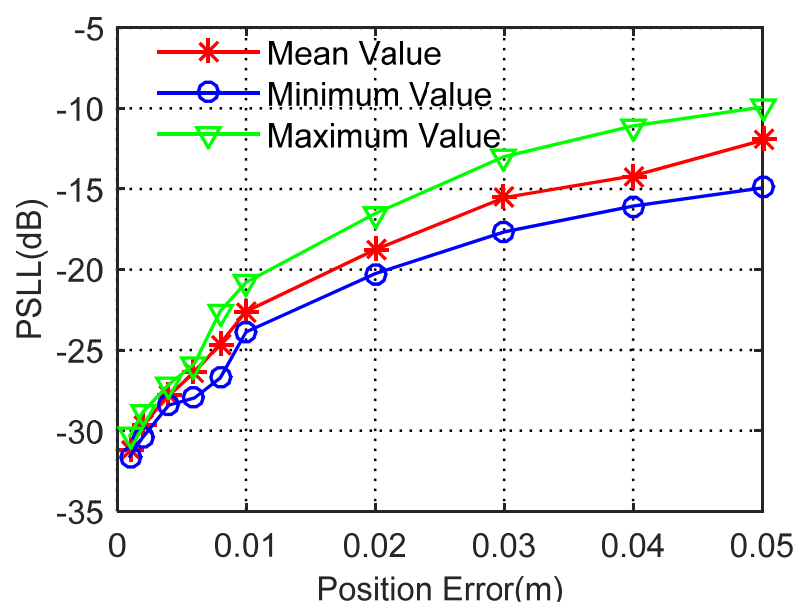

Fig. 6. PSLL change curves vs. position errors.

that the PSLL without the robust condition increases sharply with the increase of the position error of the array element, and the comprehensive performance of the pattern deteriorates. When the error position grows to $5 \mathrm{~cm}$, the maximum value of the PSLL reaches approximately $-10 \mathrm{~dB}$.

Figure 7 shows the power pattern at a position error of $0.05 \mathrm{~m}, 0.01 \mathrm{~m}, 0.006 \mathrm{~m}$, and $0.001 \mathrm{~m}$, respectively. When the position error is $1 \mathrm{~mm}$, the PSLL is as low as $-31.73 \mathrm{~dB}$, which is only $0.4 \mathrm{~dB}$ higher than the non-position error. By comparison, the PSLL of $5 \mathrm{~cm}$ uncertainty location is up to $-11.93 \mathrm{~dB}$, which illustrates the worse grating lobe suppression performance.

\subsection{Simulation of Robust Array Layout and Robust Excitation}

The previous section describes the influence of position disturbance on array manifold mismatch. In this section, we consider the array manifold mismatch error, and try to optimize robust array layout and robust weight coefficients simultaneously with PSO-RSOCP algorithm. This algorithm has been explained in detail in Sec. 3.3, in which the local optimization algorithm was solved by using the

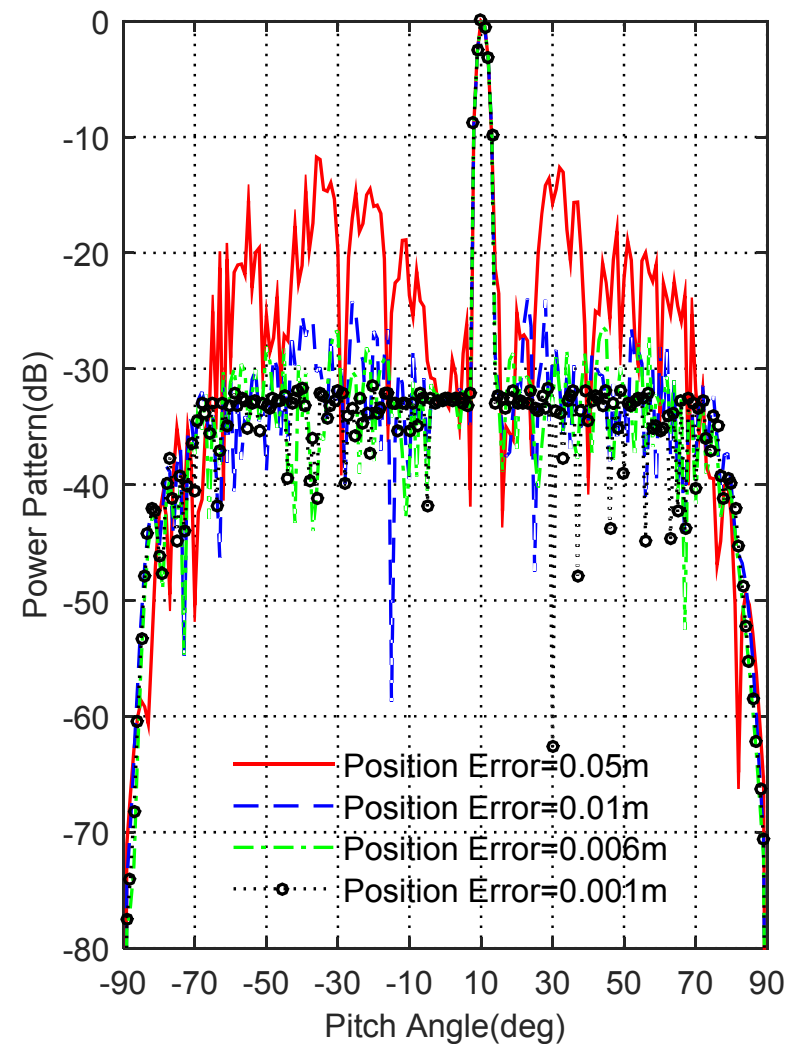

Fig. 7. Radiation patterns while different position errors.

robust convex optimization algorithm according to (9). The fitness function value of each particle in each iteration was obtained from (10). We set the position error $1 \mathrm{~cm}$. And the simulation is performed according to the obtained optimal array. The comparisons between robust and non-robust directivity patterns are shown in the same graph to display the robustness.

Figure 8 shows the radiation patterns of the optimized array with the position error $1 \mathrm{~cm}$. The radiation patterns maintain a comparatively low PSLL even when the robust constraint isn't included, which suggests that the array layout under this circumstance has a stronger tolerance against the array manifold mismatch. In other words, this optimization of the array elements is insensitive to the array errors and has the best stability for practical use.

To further discuss the validity and reliability of the PSO-RSOCP method, Figure 9 draws the PSLL change curves obtained by this algorithm for 50 independent trials. By comparison, the PSLL of the robust constraints fluctuates around $-27 \mathrm{~dB}$ while the PSLL of the ordinary optimization is up to $-24 \mathrm{~dB}$, which suggests that the robust constraints on weights and locations have a stronger ability to resist array manifold mismatch.

Figure 10 presents the array layout optimized by the PSO-RSOCP algorithm and non-robust algorithm, respectively. There is a big disparity between the two placements. From the above analysis, it is apparent that robust array layout composed of red circles has a stronger stability against array manifold mismatch while using RSOCP optimization to optimize the elements' weights in local optimization. 


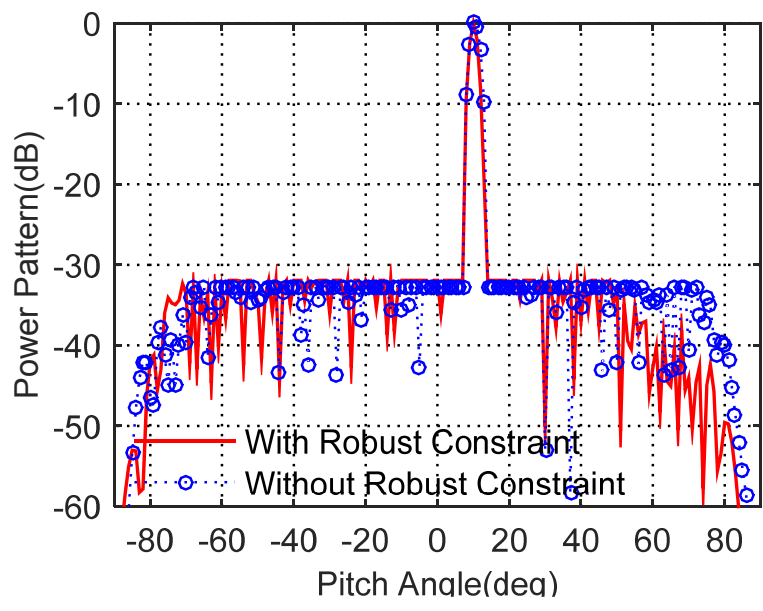

Fig. 8. Radiation patterns while position error is $1 \mathrm{~cm}$.

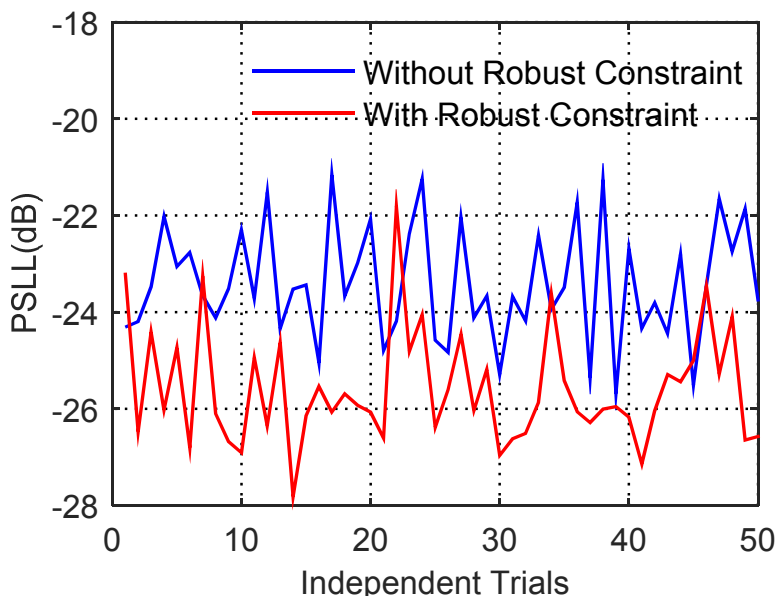

Fig. 9. PSLL change curves under robust constraint and without robust constraint vs. 50 independent trials.

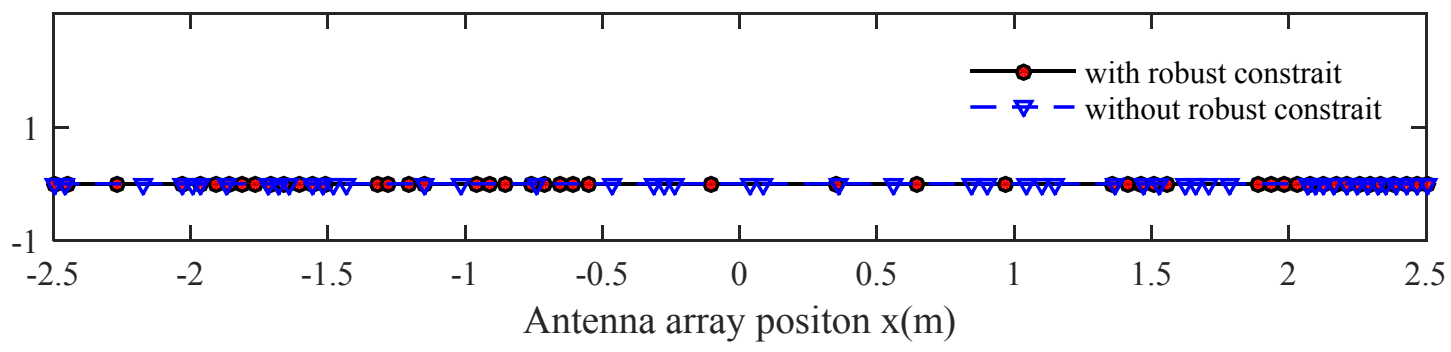

Fig. 10. Array elements' distribution while position error is $1 \mathrm{~cm}$.

\section{Conclusions}

In a sparse array, the high PSLL due to grating lobes and array manifold mismatch errors caused by array jitter and mutual coupling always influence the performance of the pattern synthesis. To solve these problems, a robust hybrid optimization method based on PSO and convex optimization is proposed for the grating lobes suppression of the sparse array in this paper, where we use PSO as a global searcher to optimize the elements' positions and convex optimization as a local searcher to optimize the elements' weight coefficients to keep the complete match of positions and weights. In addition, we introduce the array manifold errors to get a much more robust convex model. (The simulation results of sparse linear array of 50 array elements and with $70 \%$ sparse rate verify the effectiveness of the algorithm.) However, as the array errors increase, the distortions of the pattern synthesis become large. Therefore, we consider introducing robust weights optimization into the local optimization to get a more robust array layout, which can get a lower steady PSLL no matter the weight coefficients are robust or not. The robust algorithm proposed in this paper is capable of enhancing the ability of beam form optimization against array manifold mismatch and can achieve more robust excitation weights and positions, which explain its great value in practical use.

\section{Acknowledgments}

The authors would like to thank the anonymous reviewers for their comments that help to improve the quality of this article. This work was supported by the Fundamental Research Funds for the Central Universities, No. NS2016043.

\section{References}

[1] HAWES, M. B., LIU, W. Robust sparse antenna array design via compressive sensing. In Proceedings of the 18th International Conference on Digital Signal Processing (DSP). Fira (Greece), 2013, p. 1-5. DOI: 10.1109/ICDSP.2013.6622797

[2] PAPPULA, L., GHOSH, D. Sparse antenna array synthesis using multi-objective optimization. In Proceedings of the 4th IEEE Applied Electromagnetics Conference (AEMC). Bhubaneswar (India), 2013, p. 1-2. DOI: 10.1109/AEMC.2013.7045039

[3] HA, B. V., ZICH, R. E., MUSSETTA, M., et al. Synthesis of sparse planar array using modified Bayesian Optimization Algorithm. In Proceedings of the 2013 International Conference on Electromagnetics in Advanced Applications (ICEAA). Torino (Italy), 2013, p. 1541-1543. DOI: 10.1109/ICEAA.2013.6632509

[4] HA, B. V., ZICH, R. E., MUSSETTA, M., et al. Thinned array optimization by means of M-cGA. In Proceedings of the IEEE Antennas and Propagation Society International Symposium 
(APSURSI). Memphis (TN, USA), 2014. p. 1956-1957. DOI: 10.1109/APS.2014.6905305

[5] EPCACAN, E., CilOglu, T., CANDAN, C., et al. Removing grating lobes in sparse sensor arrays with a nonlinear approach. In Proceedings of the 23rd Signal Processing and Communications Applications Conference (SIU). Malatya (Turkey), 2015, p. 1950-1953. DOI: $10.1109 /$ SIU.2015.7130244

[6] DAM, H. H., RIMANTHO, D., NORDHOLM, S. E. Frequency invariant beamformer robust against array element mismatch. In Proceedings of the 9th International Conference on Information, Communications and Signal Processing (ICICS). Tainan (Taiwan), 2013, p. 1-4. DOI: 10.1109/ICICS.2013.6782891

[7] ZHAO, Y., LIU, W. Robust fixed frequency invariant beamformer design subject to norm-bounded errors. IEEE Signal Processing Letters, 2013, vol. 20, no. 2, p. 169-172. DOI: 10.1109/LSP.2012.2237028

[8] CHEN, K., ZHU, Y., NI, X., et al. Low sidelobe sparse concentric ring arrays optimization using modified GA. International Journal of Antennas and Propagation, 2015, p. 1-5. DOI: $10.1155 / 2015 / 147247$

[9] HAUPT, R. L. Optimized element spacing for low sidelobe concentric ring arrays. IEEE Transactions on Antennas and Propagation, 2008, vol. 56, no. 1, p. 266-268. DOI: 10.1109/TAP.2007.913176

[10] JIANG, Y., ZHANG, S., GUO, Q., et al. Synthesis of uniformly excited concentric ring arrays using the Improved Integer GA. IEEE Antennas and Wireless Propagation Letters, 2015, vol. 15, p. 1124-1127. DOI: 10.1109/LAWP.2015.2496173

[11] JIANG, Y., ZHANG, S. An innovative strategy for synthesis of uniformly weighted circular aperture antenna array based on the weighting density method. IEEE Antennas and Wireless Propagation Letters, 2013, vol. 12, p. 725-728. DOI: 10.1109/LAWP.2013.2264833

[12] LI, S. Grate lobes/side lobes suppression for sparse array design by using genetic algorithms. In Proceedings of the 2nd International Conference on Innovations in Bio-Inspired Computing and Applications (IBICA). Shenzhen (China), 2011, p. 371-373. DOI: 10.1109/IBICA.2011.97

[13] HAWES, M. B., LIU, W. Pattern synthesis of linear antenna arrays using a genetic algorithm with physical size constraint. In Proceedings of the 6th European Conference on Antennas and Propagation (EUCAP). Prague (Czech Republic), 2012, p. 3046-3049. DOI: 10.1109/EuCAP.2012.6206259

[14] ROCCA, P., MAILlOUX, R. J., TOSO, G. GA-based optimization of irregular subarray layouts for wideband phased arrays design. IEEE Antennas and Wireless Propagation Letters, 2015, vol. 14, p. 131-134. DOI: 10.1109/LAWP.2014.2356855

[15] DONG, W., ZENG, S., WU, Y., et al. Linear sparse arrays designed by dynamic constrained multi-objective evolutionary algorithm. In Proceedings of the IEEE Congress on Evolutionary Computation (CEC). Beijing (China), 2014, p. 3067-3072. DOI: 10.1109/CEC.2014.6900448

[16] CAO, H., JIANG, T., CHEN, X. Array optimization for MIMO radar by particle swarm algorithm. In Proceedings of the IEEE CIE International Conference on Radar (Radar). Chengdu (China), 2011, p. 99-103. DOI: 10.1109/CIERadar.2011.6159485

[17] LI, J., XU, J., ZHANG, X. Circular sparse array beam synthesis based on particle swarm optimization with consideration of polarization. In Proceedings of the IET International Radar Conference 2013. Xi'an (China), 2013, p. 0564-0564. DOI: $10.1049 /$ cp.2013.0450
[18] BERA, R., ROY, J. S. Thinning of elliptical and concentric elliptical antenna arrays using particle swarm optimization. Microwave Review, 2013, vol. 19, no. 1, p. 2-7. ISSN 1450-5835

[19] YU, D., ZHANG, Z. H., LIU, W. L. Synthesis of a plane-wave via linear array with unequal interval. In Proceedings of the 10th International Symposium on Antennas, Propagation \& EM Theory (ISAPE). Xi'an (China), 2012, p. 827-829. DOI: 10.1109/ISAPE.2012.6408899

[20] HSU, C. H., CHEN, C. H., SHYR, W. J., et al. Optimizing beam pattern of linear adaptive phase array antenna based on particle swarm optimization. In Proceedings of the Fourth International Conference on Genetic and Evolutionary Computing (ICGEC 2010). Shenzhen Univ. (China), 2010, p. 586-589. DOI: 10.1109/ICGEC.2010.150

[21] LIU, X. Z, YANG, W. L, GAO, Z. Z., et al. A new method for the synthesis of sparse linear array. In Proceedings of the 2011 IEEE CIE International Conference on Radar (Radar). Chengdu (China), 2011, p. 1139-1142. DOI: 10.1109/CIERadar.2011.6159754

[22] CHONG, E. P., ZAK, S. H. An Iintroduction to Optimization. $4^{\text {th }}$ ed. John Wiley \& Sons, 2013. ISBN: 9787121267154

[23] BOYD, S., VANDENBERGHE, L. Convex Optimization. Cambridge University Press, 2003. ISBN: 9780521833783

[24] SEDUMI. [Online]. Available at: http://sedumi.ie.lehigh.edu.

[25] GRANT, M., BOYD, S. CVX: MATLAB Software for Disciplined Convex Programming. [Online] Cited 2012-09. Available at: http://cvxr.com/cvx.

[26] FUCHS, B. Synthesis of sparse arrays with focused or shaped beampattern via sequential convex optimizations. IEEE Transactions on Antennas and Propagation, 2012, vol. 60, no. 7, p. 3499-3503. DOI: 10.1109/TAP.2012.2196951

[27] FUCHS, B., SKRIVERVIK, A. K., MOSIG, J. R. Sparse array synthesis via sequential convex optimizations. In Proceedings of the 6th European Conference on Antennas and Propagation (EUCAP). Prague (Czech Republic), 2012, p. 2216-2219. DOI: 10.1109/EuCAP.2012.6206583

[28] TU, G., CHEN, E. Sparse array synthesis based on iterative weighted $\mathrm{L}_{1}$ norm. In Proceedings of the International Conference on Computational Problem-solving (ICCP). Jiuzhai (China), 2013, p. 238-240. DOI: $10.1109 /$ ICCPS.2013.6893581

[29] PRISCO, G., D'URSO, M. Maximally sparse arrays via sequential convex optimizations. IEEE Antennas and Wireless Propagation Letters, 2012, vol. 11, p. 192-195. DOI: 10.1109/LAWP.2012.2186626

[30] LIU, X., HU, F., XIONG, W., et al. Cuboid sparse array synthesis for sensor selection by convex optimization with constrained beam pattern based on WBAN. In Proceedings of the Wireless Communications \& Signal Processing (WCSP). Nanjing (China), 2015, p. 1-5. DOI: 10.1109/WCSP.2015.7341153

[31] CHEN, H., WAN, Q. Non-uniform array pattern synthesis using reweighted $\ell$ 1-norm minimization. AEU-International Journal of Electronics and Communications, 2013, vol. 67, no. 9, p. 795-798. DOI: $10.1016 /$ j.aeue.2013.03.010

[32] ZHAO, Y., HUANG, J., ZHANG, Z. Synthesis of unequally spaced array by genetic algorithm and convex optimization. In Proceedings of the IET International Radar Conference. Guilin (China), 2009, p. 1-4. DOI: 10.1049/cp.2009.0479

[33] YUAN FEI, YANG BO, HUANG, Z. Joint optimization about pattern synthesis of circular arrays based on convex optimization and modified genetic algorithm. Fire Control and Command Control, 2015, vol. 40, no. 1, p. 58-61. 
[34] ANSELMI, N., ROCCA, P., MASSA, A., et al. Synthesis of robust beamforming weights in linear antenna arrays. In Proceedings of the Antenna Measurements \& Applications (CAMA). Antibes (France), 2014, p. 1-3. DOI: 10.1109/CAMA.2014.7003325

[35] ANSELMI, N., ROCCA, P., SALUCCI, M., et al. Optimization of excitation tolerances for robust beamforming in linear arrays. IET Microwaves, Antennas \& Propagation, 2016, vol. 10, no. 2, p. 208-214. DOI: 10.1049/iet-map.2015.0508

[36] ROCCA, P., MANICA, L., ANSELMI, N., et al. Analysis of the pattern tolerances in linear arrays with arbitrary amplitude errors. IEEE Antennas and Wireless Propagation Letters, 2013, vol. 12, no. 6, p. 639-642. DOI: 10.1109/LAWP.2013.2261912

[37] MANICA, L., ANSELMI, N., ROCCA, P., et al. Robust maskconstrained linear array synthesis through an interval-based particle SWARM optimization. IET Microwaves, Antennas and Propagation, 2013, vol. 7, no. 12, p. 976-984. DOI: 10.1049/ietmap.2013.0203

[38] ANSELMI, N., MANICA, L., ROCCA, P., et al. Synthesis of robust linear antenna arrays exploiting an interval-based particle swarm optimizer. In Proceedings of the 8th European Conference on Antennas and Propagation (EuCAP). The Hague (Netherlands), 2014, p. 2255-2258. DOI: 10.1109/EuCAP.2014.6902262

[39] HASSANiEN, A., VOROBYOV, S. A., WONG, K. M. Robust adaptive beamforming using sequential quadratic programming: An iterative solution to the mismatch problem. IEEE Signal Processing Letters, 2008, vol. 15, p. 733-736. DOI: 10.1109/LSP.2008.2001115

[40] YANG, K., ZHAO, Z., OUYANG, J., et al. Optimization method on conformal array element positions for low sidelobe pattern synthesis. IET Microwaves, Antennas and Propagation, 2012, vol. 6, no. 6, p. 646-652. DOI: 10.1049/iet-map.2011.0330

[41] KHABBAZIBASMENJ, A., VOROBYOV, S. A., HASSANIEN, A. Robust adaptive beamforming based on steering vector estimation with as little as possible prior information. IEEE Transactions on Signal Processing, 2012, vol. 60, no. 6, p. 2974-2987. DOI: 10.1109/TSP.2012.2189389

[42] YAN, S., HOVEM, J. M. Array pattern synthesis with robustness against manifold vectors uncertainty. IEEE Journal of Oceanic Engineering, 2008, vol. 33, no. 4, p. 405-413. DOI: 10.1109/JOE.2008.2002583

[43] YAN, S. Robust array pattern synthesis with uncertain manifold vector. Journal of the Acoustical Society of America, 2008, vol. 123 , no. 5. DOI: 10.1121/1.2933859

[44] LIU, J., ZHAO, Z., WANG, J., et al. A robust sparse optimization for pattern synthesis with unknown manifold error. In Proceedings of the IEEE Radar Conference. Cincinnati (OH, USA), 2014, p. 99-103. DOI: 10.1109/RADAR.2014.6875563

\section{About the Authors ...}

Hailin LI received the Ph.D. degree in Signal and Information Processing from the Nanjing University of Aeronautics and Astronautics (NUAA), Nanjing, China, in 2013. He is a lecturer in the College of Electronic and Information Engineering of NUAA. His research interests are image processing, digital signal processors, array signal processing.

Jialing LIU was born in Nanjing, China. She received the B.S. degree from Nanjing University of Aeronautics and Astronautics (NUAA) in 2017, and she is currently working toward her M.S. degree in NUAA. Her main research interests include the conformal antenna array and array synthesis.

Jie SUN was born in Xinyang, China. She received the B.S. degree from Nanjing University of Aeronautics and Astronautics (NUAA) in 2015, and she is currently working toward her M.S. degree in NUAA. Her main research interests include the conformal antenna array and array synthesis.

Aihua CAO was born in Nantong, China. She received the B.S. degree from Nantong University in 2014, and she is currently working toward her M.S. degree in Nanjing University of Aeronautics and Astronautics (NUAA). Her main research interests include conformal array and array signal processing.

Can JIN was born in Wuhan, China. She received the B.S. degree from Nanjing University of Aeronautics and Astronautics (NUAA) in 2015, and she is currently working toward her M.S. degree in NUAA. Her main research interests include the conformal antenna array and array synthesis.

Jianjiang ZHOU was born in Nantong, China. He received the M.S. degree and the Ph.D. degree from Nanjing University of Aeronautics and Astronautics (NUAA) in 1988 and 2001 respectively, and then became a professor in NUAA. His main research interests include aircraft radio frequency stealth, radar signal processing, and array signal processing. 